\title{
Team Woosh Generator 2012 WSGC Collegiate Rocket Competition
}

\author{
Milwaukee School of Engineering \\ Devin Dolby \\ James Ihrcke \\ Brandon Jackson \\ Eric Johnson \\ Kirsti Pajunen
}

\begin{abstract}
The objective of the 2012 Wisconsin Space Grant Consortium Collegiate Rocket competition was to design, build, and launch a single-stage high powered rocket that is capable of transmitting live video from a downward looking camera during its ascent. The rocket must reach a target altitude of $3000 \mathrm{ft}$ and deploy a parachute(s) electronically for a successful recovery. Upon recovery, the rocket must be determined to be in a flyable condition to be considered a successful launch. Teams receive a launch score based on their combination of reaching the desired altitude and the quality of their video received. ${ }^{1}$

After running preliminary simulations, Team Woosh Generator decided to select a Cesaroni J357 motor with a 3.0-in airframe diameter. A BoosterVision video recorder, transmitter and receiver system was selected to complete the live video feed requirement. The camera will be located on the exterior of the rocket and will be protected from drag forces during flight from a shroud. Upon reaching apogee a drogue shot will deploy under which the rocket will descend until it reaches an altitude of 500 feet. A second chute will then deploy such that a slow descent speed is obtained for landing. Redundant flight altimeters will be utilized to ensure proper chute deployment.
\end{abstract}

Included in this report are design details considered, anticipated performance, photos of the construction process, and flight results.

\footnotetext{
${ }^{1}$ WSGC 2011 Collegiate Rocket Competition Handbook
} 


\subsection{Rocket Design and Construction}

The following subsections will detail the motor selection process, airframe design, fin design, pressure relief considerations, electronics bays, and recovery method.

\subsection{Motor Selection}

The competition parameters limited the motor selection to eight Cesaroni motors ranging from I to $\mathrm{K}$ class. Teams were than challenged to select a motor which would sufficiently meet the requirements of the completion based on their design.

It was decided to further develop a MATLAB program which was utilized by team member for a previous year's competition to analyze the performance of each motor across a range of potential weights given an anticipated rocket geometry and drag coefficient. The algorithms used in this program have proved comparable to both commercially available codes along with flight results from previous launches.

The code utilized took into account the following factors when approximating rocket performance:

- Aerodynamic drag

- Mass change of the rocket due to propellant flux

- Gravitational forces

The following assumptions were made regarding the geometry of the rocket and launch conditions:

- Body tube diameter of 3.0-in

- $\mathrm{C}_{\mathrm{D}}=0.55$

- Standard temperature and pressure

- No wind

It should be noted that factors such as wind, stability, rotation, and deviation from vertical flight could not be accounted for through this simulation. As a result, estimates obtained are likely over estimates of probable flight performance. To account for this uncertainty a buffer of 300 feet was added to the desired altitude for motor selection. This buffer was decided from experience gained from previous year's competitions. Results from this simulation are presented in Figure 1: 


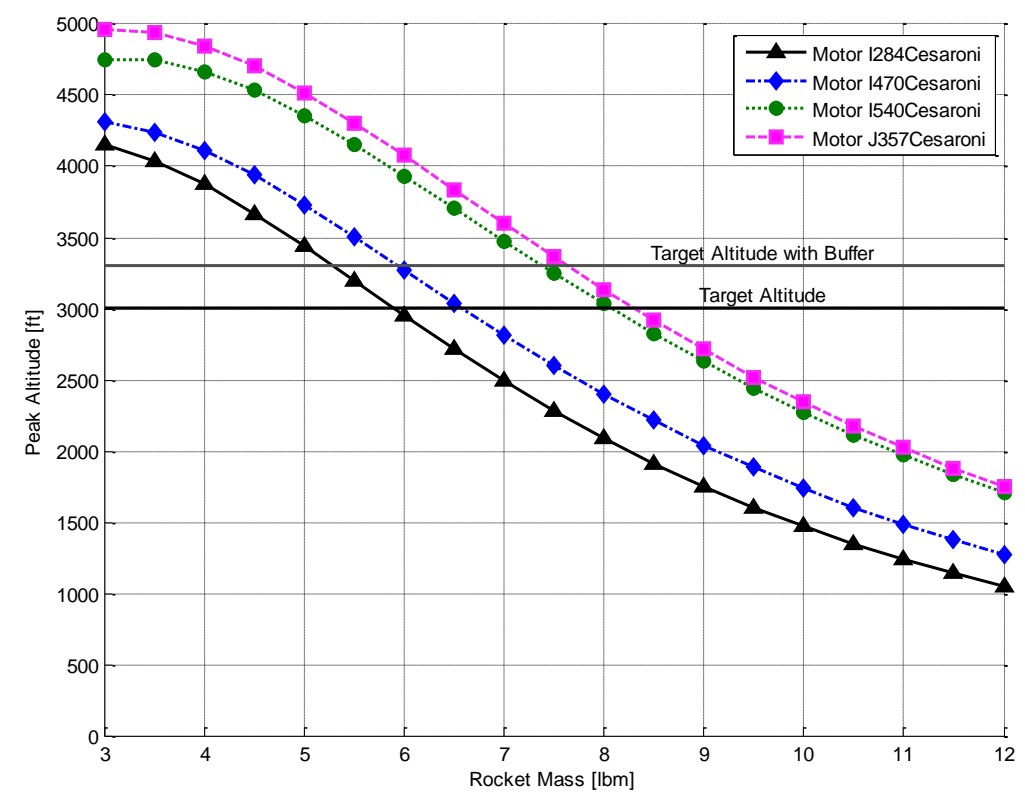

Figure 1: Analysis of rocket motor performance in predicted rocket mass range.

From this analysis it was decided to proceed in the design with the Cesaroni J357 Motor. With a projected mass between 6 and 8 lb-mass this motor is most capable of achieving the desired altitude. In the event that the constructed is less than this range small weights would allow for the rocket to achieve the predicted mass.

\subsection{Airframe Design}

Standard airframe diameters include, but are not limited to: 3.0-in, 4.0-in, and 5.5-in. Using the same simulation code discussed in Section 1.1 Motor Selection, the performance of each airframe diameter was compared. These results are presented in Figure 2:

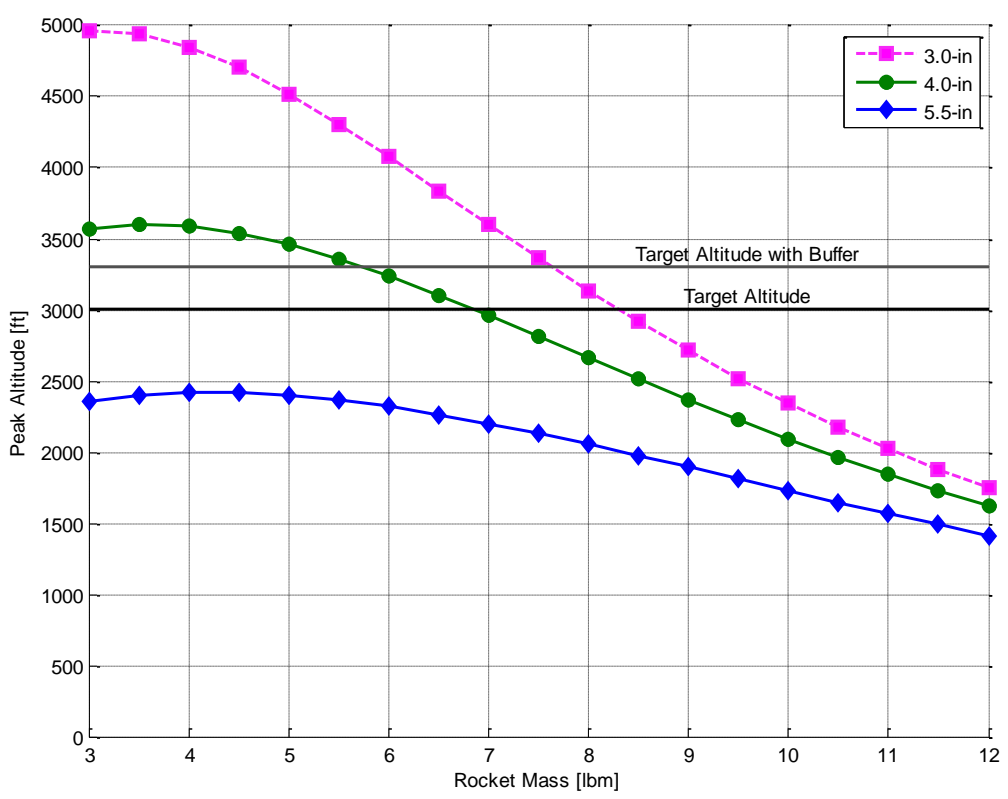

Figure 2: Analysis of airframe diameter on peak altitude in predicted rocket mass range (Cesaroni J357) 
From this analysis, it became apparent that a 3.0-in diameter body tube would be the only viable choice to meet the desired altitude.

\subsection{Center of Gravity (CG) and Center of Pressure (CP)}

The relationship between the center of pressure and center of gravity is one of the most important relationships in high powered rocketry. The center of pressure is defined as the point at which aerodynamic forces on the rocket are centered. The center of gravity is the location at which the whole weight of the rocket can be considered to act as a single force. The ratio between the locations of relative to the rocket diameter can be used to predict the stability of the rocket during flight. Generally, the center of pressure must be at least one body-tube diameter infront of the center of pressure.

The center of pressure was determined analytically for this design through the use of Barroman's theory. The results were then compared against those obtained through OpenRocket and agreed acceptably.

The following assumptions were made during the derivation of Barrowman's theory for predicting the center of pressure: ${ }^{2}$

1) The flow over the rocket is potential flow.

2) The point of the nose is sharp.

3) Fins are thin flat plates.

4) The angle of attack is near zero.

5) The flow is steady and subsonic.

6) The rocket is a rigid body.

7) The rocket is axially symmetric.

The rocket design presented in this paper did violate some of these assumptions, particularly assumptions 2, 6, and 7. However, the theory was still applied with the understanding that minor uncertainties will be present as a result.

The two centers of gravity, before and after burnout, were estimated experimentally. Sand was used to simulate the mass of the propellant and while the rocket was balanced on a wooden dowel to determine the location where it remained static.

The overall location of the center of pressure and center of gravity for the rocket are presented in Table I:

Table I: Locations of CP and CG (In Inches)*

\begin{tabular}{lccc}
\hline \hline & $\begin{array}{c}\text { Center of Gravity } \\
(\mathrm{CG})\end{array}$ & $\begin{array}{c}\text { Center of Pressure } \\
(\mathrm{CP})\end{array}$ & Stability (Caliber) \\
\hline Ignition & 49 & 54.5 & 1.77 \\
Motor Burnout & 47.25 & 54.5 & 2.33 \\
\hline \hline
\end{tabular}

* References from the nose cone tip.

\footnotetext{
${ }^{2}$ Barrowman, James. "The Theoretical Predictions of the Center of Pressure." (1966). Apogee Rockets. Web. 13 Apr. 2011. <http://www.apogeerockets.com/education/downloads/barrowman_report.pdf>.
} 
From this analysis it is projected that the rocket will be stable during the duration of the ascent portion of the flight. The rocket is currently over stable; however, prior to launch when it is in its flight configuration this process will be performed again and ballast will shifted to achieve a desired ratio.

\subsection{Electronics Bay}

The electronics bays, which serve as couplers for the bottom, middle, and top sections of the rocket, houses both the parachute altimeters in addition to: the WSGC RDAS altimeter, camera, transmitter, and batteries. To insulate the electronics from ejection-charge gases, a standard design was employed which utilized bulkheads above and below a tube coupler. Threaded steel rods were passed through the bay to which the electronics board was attached. Finally, holes were drilled in the tube coupler to allow for wires to be passed through, thus allowing the altimeters to be armed while the rocket is on the launch rail.

An image showing the front side of one of the electronics bays is shown in Figure 3. It should be noted that the components shown are not secured in their final flight configuration.

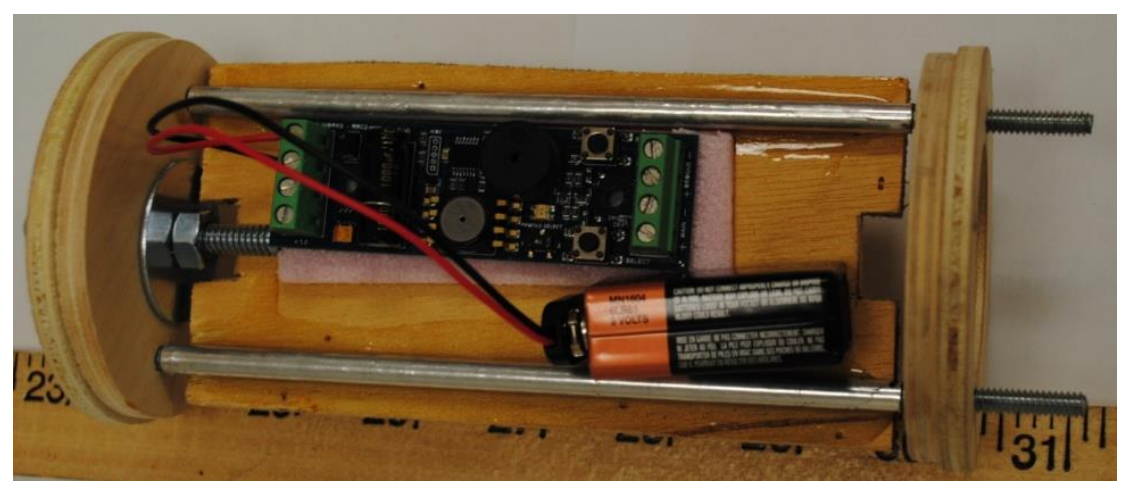

Figure 3: Lower electronics bay board.

\subsection{Recovery}

A dual deployment recovery method was selected for this design. An 18-in drogue chute will deploy at apogee and allow the rocket to descend to 500-ft where the SkyAngle Classic 44-in main chute will be deployed. For this rocket a descent rate of $20 \mathrm{ft} / \mathrm{s}$ is estimated once the main chute is deployed. Redundant RRC-2 mini altimeters are incorporated into the lower electronics bay. The location of the main and drogue chutes are shown below in Figure 4:

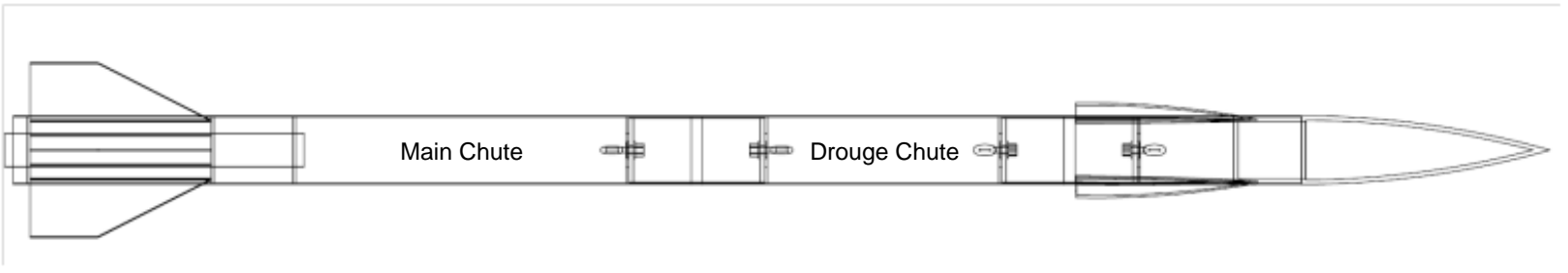

Figure 4: Full assembly with locations of parachutes

Nylon shear pins will be utilized to obtain controlled separation during descent along with keeping the nose cone attached. 


\subsection{Video Methodology}

The commercially available BoosterVision GearCam was selected to achieve the live video requirement of the competition parameters. A 1.5-in nosecone was split symmetrically and installed vertically along the side of the upper airframe section to function as a shroud for the camera, shown in Figure 5. The camera is mounted within one of these nosecone sections and oriented to achieve a downward facing image.

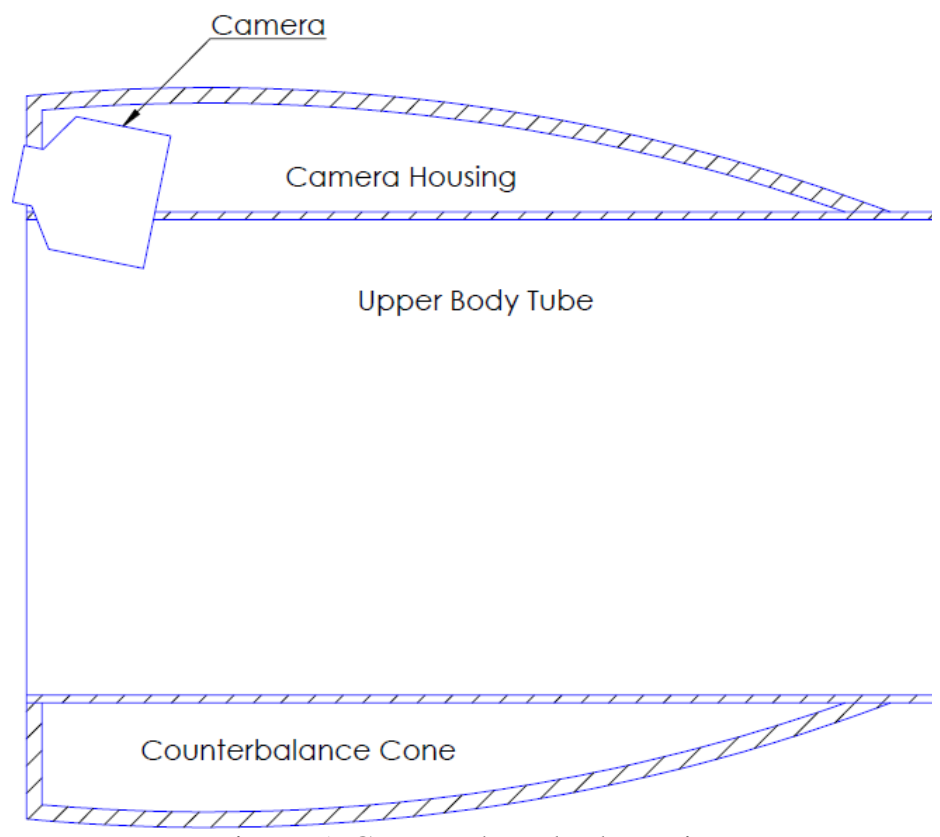

Figure 5: Camera shroud schematic

The following subsections will discuss the camera, transmitter, and receiver in further detail.

\subsection{Camera, Transmitter and Receiver}

The camera and transmitter are combined into a single functioning unit, show in Figure 6. The transmitter specifications state that video up to $5600-\mathrm{ft}$ can be achieved with the standard antenna/receiver. These numbers could not be verified experimentally on the ground since signal absorption from the ground severely decreases these numbers.

The corresponding receiver is shown in Figure 7 and allows for RCA video output into the TV tuner for recording. The black antenna shown in Figure 7 will be removed for the competition flight and replaced with a directional antenna allowing for improved reception. One of the team members will then be responsible during the flight for tracking the rocket during its accent with the antenna. 


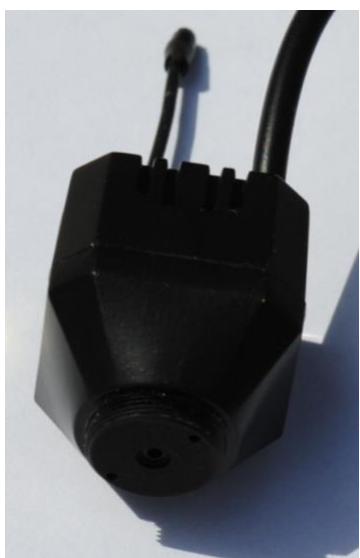

Figure 6: BoosterVision Camera and Transmitter

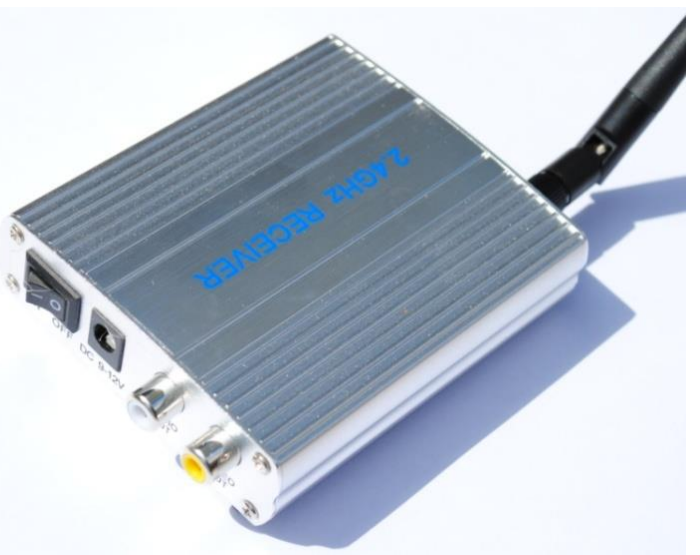

Figure 7: BoosterVision Receiver

\subsection{Anticipated Performance}

Two simulation programs were utilized to design and estimate the performance of the rocket. The programs used to simulate performance were OpenRocket and a MATLAB simulation program which was written by a team member. The results of both simulations were compared to determine the overall predicted performance of the rocket. These simulation programs will be detailed further in subsequent sections.

\subsection{MATLAB Simulation}

\subsubsection{Limitations and Assumptions}

The primary assumptions made were that the rocket would be launched vertically and that the rocket would follow a vertical flight path. Additionally, standard temperature and pressure were assumed to determine air density, which was also assumed to be constant throughout the range of flight.

\subsubsection{Numerical Methods}

The MATLAB simulation was designed to be a basic simulation program used in addition to OpenRocket. This program was originally developed and used in the 2010 and 2011 WSGC competition and compared closely with flight data. The program was designed to perform the following functions:

- Load thrust data obtained from ThrustCurve.org

- Interpolate thrust curve for more discrete steps

- Calculate change in mass resulting from burnt propellant

- Calculate velocity from the combined impulse from drag, gravity, and thrust

- Calculate altitude and acceleration from velocity

- Determine maximum altitude, velocity while leaving the launch rail, and landing velocity

- Export all data to excel for graphical analysis

The velocity of the rocket was determined from the previous momentum plus the impulse. This relationship is shown in Eq. 1: 


$$
m_{i} v_{i}+F_{i} \Delta t=m_{i+1} v_{i+1}
$$

Where $F_{i}$ is the net force acting on the rocket and $\Delta t$ is the time step between calculations. The net force acting on the rocket during accent is expressed in Eq. 2:

$$
\begin{aligned}
F_{n e t} & =F_{\text {grav }}+F_{\text {drag }}+F_{\text {thrust }} \\
& =m_{i} g+\frac{1}{2} \rho v_{i}^{2} C_{d} A+T_{i}
\end{aligned}
$$

Where:

$\rho$ is the density of air

$C_{d}$ is the coefficient of drag

$A$ is the frontal cross sectional area of the rocket

$T_{i}$ is force from the motor

Substituting Eq. 2 into Eq. 1 and solving for $v_{i+1}$ yields:

$$
v_{i+1}=\frac{1}{m_{i+1}}\left[v_{i} m_{i}+\frac{1}{\Delta t}\left(T_{i}-m_{i} g-k v_{i}^{2}\right)\right]
$$

Where:

$$
k=\frac{1}{2} C_{d} A
$$

Acceleration was calculated using Newton's second law which is expressed in Eq. 3:

$$
a_{i}=\frac{F_{i}}{m_{i}}
$$

The trapezoidal method for approximating the area under a curve was used to calculate the altitude of the rocket during the flight.

From the acceleration, velocity, and position data the maximum altitude, peak acceleration, and velocity while leaving the launch rail were determined. These results will be discussed in the subsequent section.

It should be noted that this simulation was not able to account for variables such as wind speed and direction, launch altitude, the effects of stability on flight, and flights other then perfectly vertical. 


\subsection{OpenRocket}

OpenRocket is a free, open source, software similar to RockSim. It is capable of calculating acceleration, velocity, and position data while accounting for variables including: elevation, wind speed, and the effects of individual components on performance such as: surface roughness and leading edge fin radii on drag and stability.

Also included in the program is the ability to construct full to-scale schematics of the rocket design. From this schematic the $\mathrm{CP}$ and $\mathrm{CG}$ can also be approximated.

\subsection{Flight Predictions}

The peak altitude, acceleration and velocity for both simulation methods are shown in Table II:

Table II: Simulation performance comparison

\begin{tabular}{lrr}
\hline & OpenRocket & Matlab \\
\hline Altitude $(\mathrm{ft})$ & 3302 & 3149 \\
Velocity $(\mathrm{ft} / \mathrm{s})$ & 538 & 557 \\
Acceleration $\left(\mathrm{ft} / \mathrm{s}^{\wedge} 2\right)$ & 434 & 461 \\
\hline
\end{tabular}

\subsection{Results}

Simulations were run to design and estimate flight performance of the rocket. The two programs that were used were RASAero and MATLAB code written by the team. Actual flight data was recorded using a R-DAS flight data recorder provided by WSGC. The flight of the rocket matched well with the estimates of both simulations. A comparison between predicted and measured results is shown in Table III.

Table III: Flight Performance Results

\begin{tabular}{ccc}
\hline & Max Altitude $(\mathrm{ft})$ & Max Acceleration $\left(\mathrm{ft} / \mathrm{s}^{2}\right)$ \\
\hline MATLAB & 3149 & 434 \\
Open Rocket & 3302 & 461 \\
Actual & 3314 & 425 \\
\hline & \multicolumn{2}{c}{ Percent Error From Actual } \\
\hline MATLAB & $5 \%$ & $-2 \%$ \\
Open Rocket & $0 \%$ & $-8 \%$ \\
\hline
\end{tabular}

Predicted and actual altitude and acceleration data are shown in Figure 8 and Figure 9, respectively. 


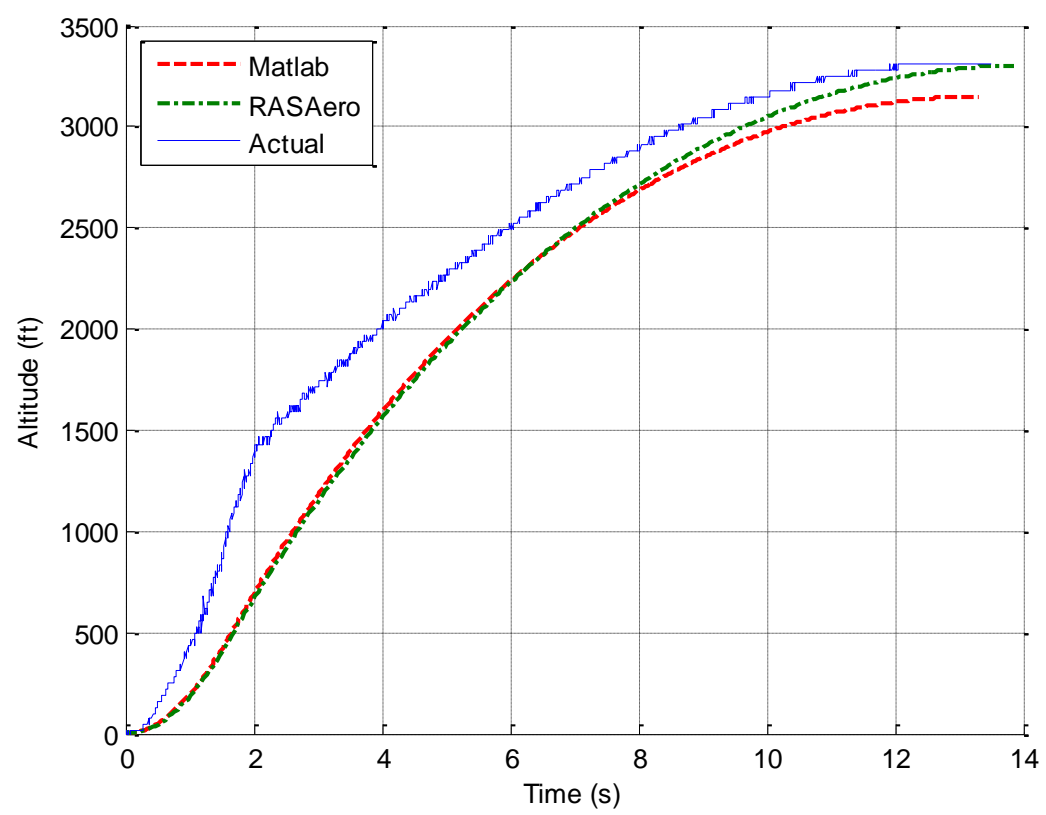

Figure 8: Comparison between Predicted and Actual Altitude

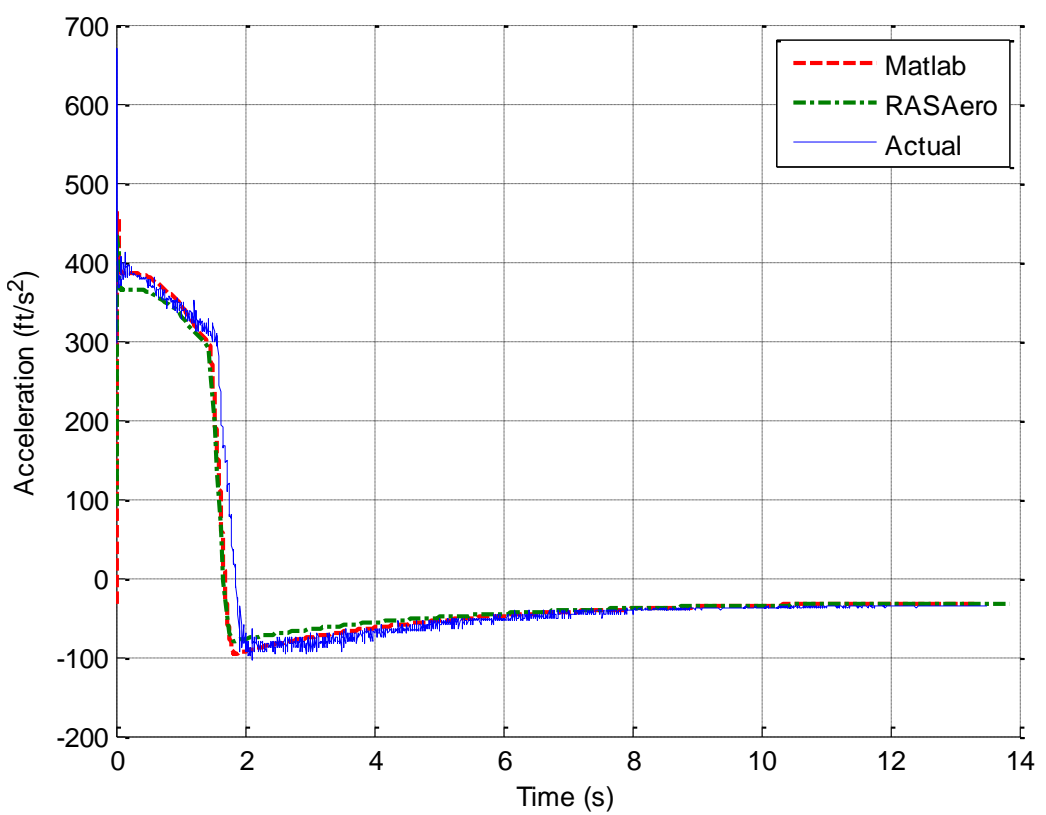

Figure 9: Comparison between Predicted and Actual Acceleration

\subsection{Conclusion}

The rocket was successfully recovered in a flyable condition in compliance with the competition rules. Performance evaluations software utilized for this design predicted the altitude and acceleration of the rocket to an exceptional margin given the uncertainties present in the launch and design. Lessons learned through this design will be incorporated into future competitions by returning team members. Thank you to the Wisconsin Space Grant Consortium for providing funding for this project and for publishing this paper in the 2012 Wisconsin Space Conference Proceedings. 